\title{
Technological advancements in head and neck free tissue transfer reconstruction
}

\author{
Syed Ahmed Ali ${ }^{1,2}$, Akina Tamaki ${ }^{1,2}$, Jason E. Thuener ${ }^{1,2}$, Shawn Li $^{1,2}$, Nicole Fowler ${ }^{1,2}$, Pierre Lavertu ${ }^{1,2}$, \\ Theodoros N. Teknos ${ }^{1,2}$, Rod P. Rezaee ${ }^{1,2}$ \\ 'Department of Otolaryngology - Head and Neck Surgery, Ear Nose and Throat Institute, University Hospitals Cleveland Medical \\ Center, Cleveland, OH 44106, USA. \\ ${ }^{2}$ Department of Otolaryngology - Head and Neck Surgery, Case Western Reserve University School of Medicine, $\mathrm{Cleveland,} \mathrm{OH}$ \\ 44106, USA. \\ Correspondence to: Dr. Rod P. Rezaee, Department of Otolaryngology - Head and Neck Surgery, Ear, Nose and Throat Institute, \\ Case Western Reserve University School of Medicine, University Hospitals Cleveland Medical Center, 11100 Euclid Avenue, LKS \\ 5045, Cleveland, OH 44106, USA. E-mail: Rod.Rezaee@UHhospitals.org
}

How to cite this article: Ali SA, Tamaki A, Thuener JE, Li S, Fowler N, Lavertu P, Teknos TN, Rezaee RP. Technological advancements in head and neck free tissue transfer reconstruction. Plast Aesthet Res 2021;8:35.

https://dx.doi.org/10.20517/2347-9264.2021.29

Received: 27 Mar 2021 First Decision: 27 Apr 2021 Revised: 9 May 2021 Accepted: 25 May 2021 First online: 2 Jun 2021

Academic Editors: Mark K. Wax, Matthew Spector Copy Editor: Xi-Jun Chen Production Editor: Xi-Jun Chen

\begin{abstract}
Free tissue transfer (FTT) is a cornerstone of head and neck reconstruction. Although rare, complications of FTT surgery can be devastating, including failed flap harvest, wound breakdown, or flap loss ultimately. Thus, modern microvascular surgeons bolster surgical and clinical expertise with a growing number of technological advances to optimize patient care and outcomes. These technologies can be applied in the preoperative, intraoperative, and postoperative period. Various preoperative imaging modalities can assist in selecting the optimal donor site and advanced perforator planning. Intraoperatively, novel technologies can assist with microvascular anastomoses, operative magnification and visualization, and assess free tissue perfusion. Postoperatively, routine clinical assessment can be augmented by a variety of adjunctive monitoring techniques designed to assess tissue health, arterial inflow and venous drainage. The overall ease and success of performing FTT can be improved by employing novel technologies at every phase of the surgical process. This article will expand upon established and upcoming technological advances and the existing literatures to support their use.
\end{abstract}


Keywords: Free tissue transfer, head and neck reconstruction, perforator mapping, technology, microvascular couplers, three-dimensional exoscope, fluorescent angiography, postoperative monitoring

\section{INTRODUCTION}

Free tissue transfer (FTT) has become a cornerstone of modern head and neck surgery, as it offers a unique means of reconstructing complicated skin, soft tissue, and osseous defects. As overall success rates continue to improve, head and neck surgeons are increasingly turning to FTT as a means of restoring form and function for patients with operative and traumatic head and neck defects ${ }^{[1]}$. Since its inception, significant advances in the operation and technological practice of the field have greatly reduced surgical duration and length of inpatient stay, two key metrics in sustaining a high-volume FTT practice ${ }^{[2]}$. In particular, the coordination of a two-team approach, the advent of the microvascular coupling device, and the exploration of easily accessible, reliable donor site options have significantly reduced operative time. As technological advancements become more widely accessible, every aspect of the care provided to head and neck cancer patients has evolved, from the preoperative planning to postoperative monitoring. This chapter will explore recent technological advancements and their impacts on the modern FTT practice. An overview of the various technologies can be found in Table 1 .

\section{PREOPERATIVE}

Preoperative planning, including flap donor site selection, is imperative to decrease operative duration and optimize patient outcomes. Planning typically starts with a complete history and physical to determine the anticipated defect and patient specific factors that will influence flap selection such as body habitus and prior surgery. Flap selection is also often drived by the surgeon's prior experience and preferred donor sites.

Certain flaps have reliable anatomy and require minimal preoperative planning. For example, a physical exam is adequate when planning to do a radial forearm, ulnar, lateral arm, or latissimus. However with the use of perforator-based flaps with variable anatomy such as the anterolateral thigh (ALT), thoracodorsal artery perforator (TDAP), and deep inferior epigastric perforator (DIEP) some surgeons find preoperative planning helpful. Studies have also shown decreased operative duration when preoperative perforator mapping is utilized ${ }^{[3,4]}$. The use of various imaging modalities in perforator mapping may be valuable in cases where the skin paddle must be thinned or split. It can also be considered in cases where larger skin paddles are required, to assist with better centering the perforator within the skin paddle and capture its respective angiosome. Here we will discuss some imaging options for perforator mapping in the preoperative setting.

\section{Computer tomography angiography}

Head and neck surgeons commonly use computer tomography angiogram (CTA) for fibula flap planning to rule out vascular disease and anatomic variants in vascular supply to the foot. This also allows for perforator mapping when designing the fibula skin paddle ${ }^{[5]}$. A lesser-used alternative to CTA for perforator mapping in the fibula free flap is magnetic resonance angiography $(\mathrm{MRA})^{[6]}$. In a comparative study, Rozen et al. ${ }^{[7]}$ found that CTA is able to identify perforators as small as $0.3 \mathrm{~mm}$, smaller than the $1 \mathrm{~mm}$ perforators detectable by advancements in MRA technology. CTA is also commonly used in DIEP flap planning by breast reconstructive surgeons where its utility has been well documented. CTA for other types of perforator flaps are less commonly used by head and neck surgeons.

Chen et al. ${ }^{[8]}$ showed significantly decreased operative duration in ALT harvest by adopting preoperative CTA. Interestingly, they did not witness a significant difference in the number of perforators identified 
Table 1. Summary of prominent head and neck microvascular free tissue transfer reconstruction technologic advancements

\begin{tabular}{|c|c|}
\hline & Brief Description \\
\hline \multicolumn{2}{|l|}{ Preoperative } \\
\hline $\begin{array}{l}\text { Computer tomography (CT) } \\
\text { angiography }\end{array}$ & Utilized CT angiograms for perforator mapping and MFTT design \\
\hline Infrared thermography & Thermal sensors used to identify perforators \\
\hline Photoacoustic tomography & Utilizes a near infrared pulse laser and ultrasound to provide 3D mapping of subcutaneous vessels \\
\hline Color duplex ultrasonography & Ultrasound used for perforator mapping \\
\hline Medical modeling* & Use of preoperative imaging in modeling, planning, and plating for MFTT reconstruction \\
\hline \multicolumn{2}{|l|}{ Intraoperative } \\
\hline Microvascular couplers & $\begin{array}{l}\text { Alternative mode of vessel anastomosis to hand-suturing. Can be used with veins and arteries, with some } \\
\text { limitations }\end{array}$ \\
\hline Three-dimensional (3D) exoscope & $\begin{array}{l}\text { 3D stereoscopic camera based viewing systems used as an alternative to operating microscopes and } \\
\text { surgical loupes }\end{array}$ \\
\hline Fluorescent angiography & $\begin{array}{l}\text { Assesses arterial perfusion and venous insufficiency of the flap through the fluorescence of the skin } \\
\text { paddle }\end{array}$ \\
\hline Osseointegrated implants* & Immediate placement of implants into osseous MFTT for dental reconstruction \\
\hline \multicolumn{2}{|l|}{ Postoperative } \\
\hline Implantable dopplers & Ultrasonic probe secured to the vascular pedicle provides real-time assessment of flow \\
\hline $\begin{array}{l}\text { Color duplex doppler } \\
\text { ultrasonography }\end{array}$ & Utilizes color doppler US to identify and trace the pedicle to assess vascular flow \\
\hline Near-infrared spectrophotoscopy & $\begin{array}{l}\text { Light source emits energy at specific near-infrared wavelengths in order to measure relative changes in } \\
\text { concentration of oxygenated and deoxygenated hemoglobin }\end{array}$ \\
\hline Laser doppler flowmetry & $\begin{array}{l}\text { Fiber optic probe secured to the skin paddle for the purpose of measuring microcirculatory changes via an } \\
\text { induced doppler shift }\end{array}$ \\
\hline $\begin{array}{l}\text { Digital infrared surface thermometer } \\
\text { monitoring }\end{array}$ & Thermometer monitors temperature changes in flap surface temperature \\
\hline
\end{tabular}

${ }^{*}$ This topic is not discussed in this manuscript as it is covered in a separate section of this edition. CT: Computer tomography; MFTT: microvascular free tissue transfer; 3D: three-dimensional.

between the CTA and non-CTA group. However, the CTA allowed them to choose the leg with more perforators or to choose septocutaneous perforators which may have contributed to decreased time of harvest. CTA also helped plan for more complex flaps that required two isolated paddles. Garvey et al..$^{[9]}$ reports a $74.1 \%$ sensitivity in identifying the presence of ALT perforators when CTA was obtained. In this dataset, CTA was better at localizing proximal perforators than distal. They also reported a $77.5 \%$ accuracy in determining a septocutaneous $v s$. musculocutaneous course of the perforator. The CTA influenced operative decision making in $37.5 \%$ of their overall cases and over half of their cases that would require two skin paddles.

CTA has been described for harvest of TDAP flaps. Mun et al. ${ }^{[10]}$ reported significantly decreased operative duration and decreased length of incision when using preoperative perforator mapping. They recommend patients to be positioned similar to the intraoperative position at the time of imaging to allow for more accurate marking of perforators at the time of surgery. Kim and Lee ${ }^{[1]}$ recommend requesting $1 \mathrm{~mm}$ cuts when obtaining imaging to better visualize small perforators. They also recommend utilizing both the coronal and axial cuts to identify perforators off the transverse and descending thoracodorsal artery ${ }^{[11]}$.

Lastly, for the vessel-depleted neck, dual-phase CT (delayed images to highlight venous vasculature) has proven useful in identifying arterial and venous targets, and even guiding the surgeon to consider a local or regional flap for reconstruction ${ }^{[12]}$. 
CTA has some disadvantages that need to be considered as well. It is expensive, exposes the patient to radiation, has contraindications in certain patient populations, and is static, therefore, limiting its ability for intraoperative use. Shen et al. ${ }^{[13]}$ have tried to address this by reporting a method of designing virtual and printed templates that can be used intraoperatively to facilitate harvest.

\section{Infrared thermography}

Infrared thermography (IT) is another technique that has been described in identifying perforators in flap reconstruction. A sensor is used to create a thermogram that identifies "hot spots" which should correlate with perforators. This technique has been described in isolation as well as in combination with CTA and ultrasound in flap planning. Xiao et al. ${ }^{[14]}$ recently reported IT in comparison to CDU in ALT flap harvest. They reported a $94.3 \%$ sensitivity and $85.7 \%$ specificity in identifying perforators. Pereira et al.$^{[15]}$ reported a much higher sensitivity and specificity of $100 \%$ and $98 \%$, respectively. One major limitation of IT is its inability to provide information about the course of the perforator. Therefore, this technique is only useful in identifying where a perforator may be located. The size and course will not be determined until it is explored intraoperatively.

\section{Photoacoustic tomography}

Photoacoustic tomography (PAT) is a 3-dimensional (3D) vascular mapping technology that has recently been used in preoperative planning for flap harvest ${ }^{[1,1,1]]}$. This technology uses energy from a near infrared pulse laser which is absorbed by hemoglobin. The red blood cells become swollen and emit ultrasonic waves which can be detected with ultrasound. This allows for 3D mapping of subcutaneous vessels without the use of contrast. This technology can be particularly useful in the harvest of perforator based ALT free flaps due to its ability to map the supra-fascial course of the vessels. Tsuge et al. ${ }^{[16]}$ showed PAT is comparable to ultrasound in the evaluation of supra-fascial perforator branching patterns and is better than ultrasound at showing oblique and horizontally oriented branches. This can be particularly useful in the process of thinning ALT flaps to expand its utility in defects requiring a thin fla $\mathrm{p}^{[18]}$. PAT is limited in its ability to examine branching patterns deep to the fascia and is also unable to distinguish arteries from veins ${ }^{[16]}$.

PAT is a newer technology still under development. One of its main limitations for clinical use is the lack of anatomical reference points precluding its intraoperative use ${ }^{[16]}$. Tsuge et al. ${ }^{[17]}$ attempted to address this in their clinical trial by developing a transparent sheet with mapping that could be sterilized and used intraoperatively. Although they were able to show correlation between mapping and intraoperative findings, further refinement will be needed to overcome errors introduced by the skin incision and retraction of tissue. Continued advancements in this technology will be needed before it is widely used for perforator mapping.

\section{Color duplex ultrasonography}

Interest in ultrasound based perforator mapping has become increasingly popular due to low cost, availability, lack of contrast/radiation, and accuracy in mapping perforators. It also has the added benefit of preoperative and intraoperative use to help facilitate decreased harvest and operative times ${ }^{[3]}$. Kehrer et al. ${ }^{[19]}$ published a step-by step guide in perforator mapping which will allow the microsurgeon to complete all mapping independent of the radiology department. Although there is certainly a learning curve, experienced microsurgeons have the advantage of intimate knowledge of the anatomy accelerating their mastery of ultrasound techniques. Ultrasound can be applied to all perforator based flaps allowing for more complex flap design. 
A recent study by González Martínez et al. ${ }^{[20]}$ compared handheld Doppler, CTA, and color duplex ultrasound (CDU). CDU and CTA had a 100\% positive predictive value (PPV) for perforator identification compared to $88.6 \%$ for the handheld Doppler. The negative predictive value for CDU, CTA and Doppler was $100 \%, 94.3 \%$ and $90.56 \%$, respectively. The majority of flaps in this study were ALT but other extremity flaps were included. A systematic review by Cheng et al. ${ }^{[21]}$ has pooled data which included 54 ALT flaps showing a 94.3\% PPV. Another study by Thomas et al. ${ }^{[3]}$ compared CDU with Doppler in ALT flap harvest. They showed a significant increase in septocutaneous perforator dissection which translated to nearly a 90 min reduction in harvest time.

While CDU has the steepest learning curve of the techniques discussed, it is noninvasive, inexpensive, has no contraindications, provides detailed anatomic information, and can be adopted in any type of flap harvest.

\section{INTRAOPERATIVE}

Contributors to successful FTT include precise surgical dissection and assessment of the harvested flap for viability. Although experience and clinical judgement are irreplaceable tenets in microvascular surgery, there are numerous technologies that can be applied intraoperatively for increased success and efficiency. These technologies include the use of microvascular couplers in anastomoses, alternative and improved microscopic and operative visualization, and technology to assess flap perfusion.

\section{Microvascular couplers}

Failure of the microvascular anastomosis is one of the primary reasons for FTT loss. Therefore, any technology to improve this process is valuable. The ideal technique would minimize ischemia time, avoid vessel trauma, maintain vessel patency, and be easy to perform. Historically, arterial and venous anastomoses were performed via hand-suturing. Suturing technique certainly requires training due to the potential for back-wall placement of sutures and poor suture placement leading to thrombosis, stenosis, or anastomotic leaks. The conceptual design of the microvascular coupler was first described in 1962 by Nakayama et al. ${ }^{[22]}$ and was introduced into use in 1986 by Ostrup and Berggren ${ }^{[23]}$. The coupler design consists of two rings with interlocking pins and corresponding holes. Each end of the vessel is passed through the ring, pinned, and anastomosed together. Available couplers range from a size of 1.0 to $4.0 \mathrm{~mm}$ (Synovis Micro Companies Alliance, Birmingham, AL).

Since the introduction of this technology, venous couplers have become widely accepted and utilized in FTT. In a recent meta-analysis comparing outcomes for microvascular couplers to hand-sutured venous anastomosis, microvascular couplers were found to be safe and effective. This study was not specific to head and neck reconstruction, which represented $19 \%$ of the cases. The meta-analysis of 12,304 patients showed a thrombosis rate of $1.47 \%$ with couplers, which was not significantly different in risk of venous thrombosis compared to hand-sutured anastomosis. There was also a significant decreased anastomotic time and postoperative flap failure risk ${ }^{[24]}$. Multiple other studies have also reported consistent positive outcomes with the use of venous couplers, including those specific to head and neck reconstruction ${ }^{[25-28]}$. In a manuscript focusing on the time saved with use of microvascular couplers, Rozen et al. ${ }^{[2]]}$ concluded a significant reduction in venous anastomotic time when coupling ( $4 \mathrm{~min}$ ) compared to suturing $(22 \mathrm{~min})$ without an increase in complications. In situations where end-to-side coupling is required (typically to the internal jugular vein stump or vein proper), DeLacure et al ${ }^{[30]}$ found that they were able to successfully complete the venous anastomosis via the coupler in 33 of 37 (89\%) attempted cases. While the authors did find additional challenges in coupling to a previously radiated or dissected vessel, with technical modifications they were able to largely avoid having to convert to suture technique. 
There are criticisms for the use of microvascular couplers, the most significant being the additional cost in using this technology. The cost is higher than that of microvascular sutures, however, this may be mitigated by the operation time saved when utilizing a coupler device. A study exploring the economic cost of microvascular couplers found that the coupler device increased the initial cost by $\$ 284.40$. However, when the reduction of operative time (16.9 min) was factored, $\$ 234.89$ of savings was estimated with each coupler anastamosis $^{[31]}$.

The use of microvascular coupler for the artery has been more controversial. Arteries can be technically challenging to couple due to the lack of pliability and difficulty everting the arterial tissue over the pins. Arteries have thicker walls and may be affected by atherosclerotic disease and radiation induced thrombosis in patients with prior radiation to the field. Earlier literature on microvascular coupling of the artery has reported concerns for increased thrombosis rates ${ }^{[32-34]}$. However, recent studies have provided more support for arterial coupling. In a systematic review of microvascular couplers used in arterial anastomosis, Gundale et al ${ }^{[35]}$ reported good success rates with low rates of thrombosis. In this study, the rate of thrombosis with arterial coupling was reported to be $1.9 \%$. Additionally, computational fluid dynamic models have shown a more thrombogenic profile in a sutured anastomosis when compared to a coupled vessel $^{[36]}$.

There are novel devices engineered to assist with microvascular arterial coupling. The vessel everter device assists with securing the arterial wall to the coupler pin. As mentioned earlier, the thicker walls of the artery makes it difficult to sequentially couple the arterial vessel wall over the pins. The everter device helps to overcome this challenge by simultaneously everting and securing the arterial wall over the coupler pins ${ }^{[37]}$. This vessel coupler is used with the microvascular coupler. It is indicated for coupler sizes 2.0 to $4.0 \mathrm{~mm}$ and not recommended for smaller sizes. It is also contraindicated in patients with silicone allergies. The instrument is shaped like a pen with a silicone tip that tapers distally. The tip is designed to fit into the end of the artery and compress the arterial wall and silicone elastomer to the engaged pins. The instrument is then withdrawn leaving the vessel wall engaged onto the pins. Preliminary animal studies using the device have shown successful arterial anastomosis more easily and efficiently with comparable outcomes to handsuturing ${ }^{[37]}$. This device is currently undergoing evaluation for commercial availability (Synovis Micro Companies Alliance, Birmingham, AL).

There are several scenarios where microsurgeons should be cautious in utilizing arterial couplers. This includes situations of significant atherosclerotic or radiated vessel where intimal injury can result from eversion onto a coupler pin. In addition, some literature warns against coupling arteries where there is significant vessel mismatch (greater than 1.5:1 diameter mismatch) and arteries less than $1.5 \mathrm{~mm}$ in diameter ${ }^{[3,39]}$. The preference at our institution is to use the microvascular coupler for the vein and handsuture the artery. However, we have found situations where the arterial coupler is valuable including in salvage settings where initial hand-suturing resulted in arterial anastomotic thrombosis ${ }^{[40]}$.

\section{Three-dimensional exoscope}

Magnification is necessary for FTT and has been traditionally accomplished through the use of operating microscopes or surgical loupes. The 3D stereoscopic camera based viewing systems are the newest technology allowing for such magnification. This technology is also referred to as extracorporal telescopes, or exoscopes. The system consists of a compact high definition 3D camera placed approximately 20 to $50 \mathrm{~cm}$ above the surgical field. A sterile cover is placed over the $3 \mathrm{D}$ camera and controller allowing the surgeon to make adjustments in position, zoom, and focus. The real-time image is projected onto one or more $3 \mathrm{D}$ high-resolution monitors placed in front of the surgeon. $3 \mathrm{D}$ polarization glasses are worn by the 
operators to provide depth to the image $\mathrm{e}^{[4]}$. The exoscope can be used during dissection and/or during the microvascular anastomosis.

There are several commercially available systems including the VITOM (Karl Storz, Tuttlingen, Germany), ORBEYE (Olympus, Tokyo, Japan), Modus V (Synaptive Medical, Toronto, Canada), and KINEVO (Carl Zeiss AG, Oberkochen, Germany) exoscope ${ }^{[42]}$. The majority of the literature on exoscope use in head and neck microvascular surgery utilizes the VITOM and ORBEYE system. There are differences between the platforms. VITOM is essentially a high-resolution endoscope mounted on a pneumatic arm. The ORBEYE and Modus V are free standing exoscopes - the ORBEYE offers 3D imaging while the Modus V provides a 2-dimentional (2D) image. Finally, the KINEVO is an operating microscope that can be converted into an $\operatorname{exoscope}^{[42]}$.

This technology has been more widely utilized in neurosurgery and less so in head and neck microvascular surgery. Microvascular anastomosis using the 3D exoscope has been demonstrated in animal and laboratory simulation studies ${ }^{[1,43]}$ and, more recently, in clinical application. In 2012, Cheng et al.$^{[4]}$ reported the use of a 3D stereoscopic monitor system, in conjunction with the conventional microscope, in FTT microvascular anastomosis. In 2017, Piatkowski et al. ${ }^{[45]}$ successfully performed breast FTT reconstruction using just the 3D exoscope. Since then, multiple studies have shown that 3D exoscopes can be safely and successfully used in FTT microvascular anastomosis ${ }^{[46-49]}$. Furthermore, subjective advantages include favorable ergonomics, excellent image quality, and ease of intraoperative use $\mathrm{e}^{[50]}$.

There are several advantages of the exoscope over the conventional operating microscopes. One of the major reported advantages of the exoscopes is superior ergonomics. Chronic neck and back problems can result from neck flexion to position appropriately to ocular lenses or surgical loupes. Users of the exoscope have noted improved ergonomics and minimized need to adjust posture and position during use ${ }^{[43,50]}$. Ultimately, improved surgeon ergonomics may reduce surgeon long term disability and increase career longevity ${ }^{[50]}$. The operating microscope also has a larger footprint occupying more space in the operating room (OR) when compared to the generally more compact exoscope. The exoscope is also valuable for teaching purposes. The exoscope allows surgical trainees and assistants to have the same visualization as the senior surgeon. With the traditional operative microscopes, all other members of the surgical team, aside from the senior surgeon, may have a $2 \mathrm{D}$ or inferior view. Studies have also noted that procedures on the exoscope are easy to learn, and simulation studies have shown progressive improvement in performance with repetition ${ }^{[43]}$.

Disadvantages of the exoscope include the low depth of field and loss of resolution at higher magnifications ${ }^{[45]}$. There is debate on whether the image quality may be inferior to that of an operating microscope ${ }^{[45]}$, although other studies note superior clarity and sharpness with the exoscope $\mathrm{e}^{[41]}$. In addition, there were surgeon complaints of headaches and nausea with the earlier versions of the $3 \mathrm{D}$ optics. This issue seems to be much improved with newer versions of these exoscopes ${ }^{[42]}$. Also, in several small studies, microvascular anastomosis using the exoscope did take longer compared to that using the operative microscope ${ }^{[44,45]}$. However, a recent pilot study by Ahmad et al. ${ }^{\left[{ }^{[0]}\right]}$ showed no difference in operative time. In addition, there was no difference in ischemia time or microsurgical complications between the exoscope and traditional microscope group.

Another necessary consideration is the cost of this technology. The estimated cost of the two most commonly used exoscope systems in head and neck microvascular surgery, the VITOM and ORBEYE, is $\$ 200,000$ and $\$ 400,000$, respectively ${ }^{[42]}$. The cost accounts for multiple components including the exoscope 
camera, scope holder, $4 \mathrm{~K}$ high definition monitor, and 3D glasses. In comparison, operating microscopes can vary in cost, with start-up ranges from approximately $\$ 250,000$ to over $\$ 500,000^{[50]}$. A justification for this cost may be the utility of this technology by multiple surgical specialties, ability to use in both dissection and microvascular anastomosis, and improved ergonomics for the operative surgeon to increase overall career longevity.

In our institution, we have trialed the exoscope technology, but have not utilized this in routine practice. The operative microscope continues to be our standard of practice. There are various technologies that can provide comparable magnification during FTT; the exoscope may have advantages as far as ergonomics.

\section{Fluorescent angiography}

Fluorescent angiography using indocyanine green (ICG) can be an excellent resource in assessing flap vascular perfusion. ICG is a fluorescent dye that is administered intravenously and remains in the intravascular compartment. After injection, the dye can perfuse through vascular pedicles into the subdermal and subcutaneous plexus. It has an absorption spectrum of $800 \mathrm{~nm}$ and can be detected using an infrared camera to confirm adequate flap perfusion. Historically, it was used to measure hepatic and cardiac function but has been widely adopted for use in ophthalmology and microvascular surgery ${ }^{[51]}$.

There are multiple companies that utilize this technology: IC View (Pulsion Medical Systems, Munich, Germany), PDE (Hamamatsu Photonics, Shizuoka, Japan), Quest Spectrum (Quest, Middenmeer, Netherlands), among many others. At our institution, we use the SPY Elite (Stryker, Kalamazoos, MI), which is a standalone camera system mounted onto a workstation for video output and data storage. There are also portable handheld models as well as systems integrated into operating microscopes that work via an infrared camera (Leica, Wetzlar, Germany) ${ }^{[52]}$. Typically, ICG is stored in powder form. This is reconstituted in saline or sterile water and given through a rapid intravenous bolus. For free flap imaging, we typically use a standard dose of $10 \mathrm{mg}$. After injection, the camera system is directed into the area of interest and perfusion can be assessed.

ICG has a well-established safety profile. In a large study from 1994, Hope-Ross et al. ${ }^{[53]}$ examined 1923 tests in 1225 patients and found an incidence of $0.15 \%, 0.2 \%$, and $0.05 \%$ for mild, moderate, and severe reactions, respectively. No deaths were noted. While rare cases of anaphylaxis have been described ${ }^{[54]}$, intraoperative use should be quite safe given that the patient remains under monitored anesthesia care for an extended time following exposure. Caution should be taken in patients with iodine allergy, pregnancy, liver disease, uremia, and previous anaphylaxis to intravenous dy $\mathrm{e}^{[53]}$. ICG is metabolized by the liver and has a short halflife of 3 to $4 \mathrm{~min}$. The rapid turnover allows for repeated use throughout the same case, if necessary.

Fluorescence angiography has multiple applications in free flap surgery. It can be used to assess arterial perfusion of the flap through the overall fluorescence of the skin paddle. This gives confirmation that the arterial anastomosis is patent, and that perforators have been preserved. It can also be used to look for relative venous insufficiency by comparing the flap to surrounding native tissue. Oversaturation can suggest some level of venous congestion. Finally, it is a useful adjunct in determining the pattern of perforator distribution. This allows the surgeon to split the skin paddle safely as well as discard distal areas of skin that may have suboptimal blood supply ${ }^{[53]}$.

Some surgeons use ICG angiography routinely in every FTT. At our institution, we have found clinical and visual assessment to be adequate for most cases. We reserve the SPY for use in complex cases involving large skin paddles, multiple perforators, or if there is a clinical concern for inadequate perfusion. 


\section{POSTOPERATIVE}

While overall success rates for FTT exceeds 95\%, the need for revision surgery remains significant at 10\% to $12 \%{ }^{[55]}$. Timeliness is particularly important in identifying a potential issue with a FTT, as there exists a finite window where the flap can be salvaged while under-perfused or congested. While clinical assessment by an experienced microvascular surgeon is the gold standard, it is not practical to have this be the only measure of flap monitoring during the critical 48 to $72 \mathrm{~h}$ window where there is greatest risk for arterial or venous thrombosis. As such, the need for a sensitive and specific adjunct flap monitoring technique is crucial, particularly in the era of strict resident duty hour monitoring and increasing turnover of nursing staff. These adjunct techniques serve to complement a basic clinical examination and provide objective data with which microvascular surgeons can assess the need for possible revision surgery. The techniques vary greatly in terms of ease of use, cost of implementation, and overall specificity and sensitivity. This section will explore the basics of newer technologies in adjunct free flap vascular monitoring, as well as their advantages and disadvantages.

\section{Implantable dopplers (arterial and venous)}

The implantable doppler technology consists of a 20-MHz pulsed ultrasonic probe within a silicone cuff that can be secured to the vascular pedicle (either artery or vein) via sutures, surgical clips, or tissue sealants ${ }^{[5]}$. The potential advantage of this technology is that it provides real-time assessment of flow through the vascular pedicle and can alert clinicians to alteration of arterial flow or compromise of venous drainage in advance of clinically detectable changes in the free flap. The disadvantage largely relates to over-reliance on the technology in lieu of clinical assessment, leading to a potentially significant false-positive rate in the event of device dislodgement or failure. A meta-analysis of 22 articles comparing post-operative outcomes between an implantable doppler group and a clinical assessment group found a significantly lower free flap failure rate $(2.11 \%$ vs. $4.21 \%)$ and an improved flap salvage rate $(83 \% v \text { s. } 59 \%)^{[57]}$.

At our institution, we utilize implantable dopplers for the majority of our mucosal reconstructions. We have found that continuous, real-time monitoring in the OR after microvascular anastomosis has allowed us to identify alterations in flap perfusion related to fluctuations in the patient's blood pressure. This helps establish a minimum blood pressure goal for the anesthesiologist to titrate toward to maintain ideal flap perfusion. Specifically, if there is an alteration or loss of signal correlated to a decrease in blood pressure, we will titrate the blood pressure up to establish a minimum perfusion goal. Furthermore, the continuous monitoring while completing inset or closing the neck wounds provides an additional safeguard against unfavorable pedicle positioning, potential compression with skin flaps, or alterations in flow secondary to placement of a suction drain. Postoperatively, we have found that it provides reliable monitoring of flow through the arterial anastomosis, however we do not rely on it for assessment of the venous pedicle.

A study by Swartz et al. ${ }^{[58]}$ demonstrated that relying on an arterial probe to detect downstream venous thromboses led to an increased rate of false negatives; further laboratory analysis demonstrated that an arterial probe required a mean $220 \pm 40 \mathrm{~min}$ to detect venous thromboses, compared to a mean $6.08 \pm 2.4 \mathrm{~min}$ for a venous probe to detect venous thromboses. The caveat with this, however, is that utilization of venous probes can lead to a higher false-positive rate (up to 33\%), secondary to probe dislodgement, fibrin coating, or global device malfunction ${ }^{[57]}$.

The implantable doppler has proven itself to be a useful adjunct in the overall assessment of free flaps postoperatively, particularly for monitoring of flap perfusion where there is evidence for earlier time to flap salvage and resultant improved overall outcomes ${ }^{[59]}$. It is additionally useful for buried free flaps, providing continuous and reliable monitoring. Effective utilization does require troubleshooting pedicle geometry, 
particular in crowded anatomic spaces in the head and neck, as well as overall caregiver consciousness of protecting the external segment of the monitoring probe. Future upgrades may include more widespread use wireless monitoring capabilities, potentially reducing existing issues with probe dislodgement.

\section{Color duplex doppler ultrasonography}

Color doppler ultrasound is a non-invasive, cheap, and repeatable tool that can be used to assess arterial and venous flow as an adjunct for overall FTT assessment. Studies have typically described utilizing a 5 or $5.5 \mathrm{MHz}$ linear transducer ultrasound probe with a real-time high resolution ultrasonography machine ${ }^{[60]}$. Knowledge of the location of the pedicle (denoted by a stitch or marker) allows the user to perform realtime ultrasonography to identify and trace the pedicle from its origin in the free flap to its course into the recipient vessel. From a flap monitoring perspective, the proposed advantages of this technology include the ability to monitor buried or covered flaps, assess the health of the artery and vein in parallel to one another, and assist with assessing the exact site of obstruction in the event of a geometrical issue or an occluded vesse $^{[60-62]}$. A case series discussion by Seres et al ${ }^{[60]}$ demonstrates how color ultrasonography can provide additional information when venous congestion is of concern, particularly in cases of hematomas or free flap swelling. In all three of their described cases, the authors were able to avoid returning to the OR by confirming adequate flow through the vein via color doppler ultrasound. Further studies have found a role for color ultrasonography as a means of evaluating the loss of implantable Doppler probe signals in buried free flaps, where clinical assessment cannot distinguish between device malfunction and true flap compromise ${ }^{[62]}$.

Limitations of color Doppler ultrasonography relate to the overall cost of owning the machine and degree of knowledge and skill required to perform the imaging and accurately assess the flow through the pedicle ${ }^{[61]}$. While it is likely not feasible to train nursing staff in performing ultrasonography as part of FTT assessment, as ultrasound use becomes more widespread overall, a greater proportion of residents are becoming familiar with its use. Additionally, as ultrasound technology becomes increasingly mobile where touch-screen devices and personal cellphones are being utilized as the screens, the need for transporting larger and more costly equipment will decrease.

At our center, we do not currently utilize color ultrasonography as part of routine free flap assessment. However, as the technology becomes increasingly portable and the imaging techniques become more ingrained within the training curriculum, we do feel that it has an important role as an adjunctive measure for flap monitoring, particularly for re-operative decision making.

\section{Near-infrared spectrophotoscopy}

Near-infrared spectrophotoscopy (NIRS) technology employs a light source to emit energy at specific nearinfrared wavelengths in order to measure relative changes in concentration of oxygenated and deoxygenated hemoglobin ${ }^{[63]}$. The spectrometer typically measures relative changes in tissue concentrations of hemoglobin and presents the findings as an alteration in percentage of saturated hemoglobin $\left(\mathrm{StO}_{2}\right)$. This requires attachment of a probe to the patient's free flap, with the use of a light-blocking shield to prevent ambient light intrusion, typically placed at the time of initial surgery ${ }^{[63]}$. The surgical team must then decide a threshold value where the spectrometer will alert the clinician, typically an absolute reading of $\mathrm{StO}_{2}$ below $30 \%$ to $50 \%$ or a certain drop rate percentage over a set amount of time ${ }^{[64]}$. Proposed advantages of this adjunctive technology include its overall reliability, noninvasive nature, and ability to distinguish the source of the vascular crisis ${ }^{[6]}$. A systematic review demonstrated an improvement in surgical salvage rate and flap failure rate between NIRS monitored groups and those that did not use NIRS ${ }^{[64]}$. Disadvantages associated with NIRS system include inconsistency in alarm threshold values, potential need for repositioning of the spectrometer sensors, retail price of the sensor and the spectrometer, and inability to be used for buried 
flaps or flaps with a small cutaneous component.

With the head and neck FTT practice at our institution, given the number of free flaps that are mucosal, that have small monitoring paddles, or that are entirely buried, we have not found it practical to invest in this technology. Its application, in our estimation, is most useful for flaps with large cutaneous surfaces that are easily accessible.

\section{Laser doppler flowmetry}

Laser doppler flowmetry (LDF) is a non-invasive technology that allows for continuous monitoring of vascular perfusion. Briefly, the device consists of a specialized fiber optic probe that is secured to the cutaneous surface of a FTT for the purpose of measuring microcirculatory changes via an induced doppler shift $^{[66]}$. Centers that utilize LDF typically describe a 48 to $72 \mathrm{~h}$ monitoring window where nursing staff or residents record flow values and notify the microvascular surgeons if there is a predetermined decrease in flow over a particular period of time ${ }^{[67]}$. Studies have determined that the flowmeter is able to output value changes indicative of flap compromise 1 to $3 \mathrm{~h}$ ahead of a change in the clinical appearance of the flap, thereby decreasing the time to revision ${ }^{[68]}$. They additionally report on a low or non-existent false positive rate $^{[67,68]}$. Proponents of the LDF system cite its reliability, accuracy, and negligible overall false-positive and false-negative rates ${ }^{[6]}$. Disadvantages of the system include complexities in data interpretation, particularly between arterial and venous thromboses, technical issues if affixed to a dynamic free flap, inability to monitor buried flaps, and overall cost of adopting the system ${ }^{[69]}$. At our institution, we have not utilized LDF as an adjunct in free tissue monitoring.

\section{Digital infrared surface thermometer monitoring}

Gross temperature assessment via palpation has long been a principle of overall clinical flap monitoring. Early proponents attempted to assess for temperature change via surface temperature monitoring. Kannan ${ }^{[70]}$ were able to demonstrate a positive correlation between flap microcirculation and flap surface temperature, although this was less reliable for venous thrombosis in comparison to arterial thrombosis. A study by Khouri and Shaw ${ }^{[7]]}$ demonstrated a sensitivity of $98 \%$ and a predictive value of $75 \%$ in identifying a microvascular thrombosis. While surface temperature recordings are naturally affected by normal skin temperature fluctuations, a previous case series by Baudet et al. ${ }^{[72]}$ noted that an acute drop in temperature of $3{ }^{\circ} \mathrm{C}$ or more was found to be correlated with arterial compromise. With advances in technology, some investigators who rely on temperature changes as a means for flap monitoring have begun utilizing noncontact infrared thermometers in place of surface contact thermometers. A retrospective review of free flap failures by Papillion et al. ${ }^{[73]}$ demonstrated a temperature difference between flaps that survived and those that were ultimately unable to be salvaged. Specifically, the authors found a temperature difference of 2.6 to $5.4{ }^{\circ} \mathrm{C}$ between the FTT tissue and adjacent skin in the failing flap group ${ }^{[73]}$. Additional studies have been further able to correlate alterations in flap temperature obtained via a digital infrared thermometer to changes in capillary blood flow and hemoglobin oxygenation ${ }^{[7]}$. Clear advantages of this adjunct include ease of use, lack of reliance on contact technology that could malfunction or become dislodged, relative accuracy, and low overall adoption cost. Potential disadvantages include lack of standardized alarm thresholds, a difficulty in accounting for natural surface temperature fluctuations or those fluctuations associated with a systemic process, and that temperature change may potentially be a late finding in the overall picture of flap compromise ${ }^{[73]}$. At our institution, our residents and nursing staff are taught to evaluate for gross temperature changes as part of their clinical assessment, however we do not formally utilize surface contact thermometry or infrared thermometry as part of our adjunctive monitoring measures. 


\section{DISCUSSION}

This review article references and discusses many of the commonly implemented and upcoming technologies that has been utilized in the field of FTT today. While there are proponents to each of these advances - be it more detailed preoperative imaging modalities, improved microsurgical optics, or more sensitive postoperative adjuncts - they must be weighed against the burgeoning costs of healthcare delivery. Adoption of new technology can be exciting, however a responsible practice needs to inform their decision with objective improvement in patient outcomes and/or decrease in operative timing as it pertains to their specific institution. Furthermore, cost saving via reducing operative time needs to be carefully assessed on an individual institutional level, as a minor decrease may not necessarily translate to an appreciable reduction in operative costs.

\section{CONCLUSION}

There is a significant amount of technology that can be applied in the preoperative, intraoperative, and postoperative setting to further optimize FTT outcomes and efficiency. These technologies continue to evolve and is an exciting area of research. It is imperative for head and neck surgeons to be aware of these technological advances and apply them to clinical practice as seen fit and as local resources and practices allow.

\section{DECLARATIONS}

\section{Authors' contributions}

Made substantial contributions to conception and design of the study and performed data analysis and interpretation: Ali SA, Tamaki A, Thuener JE, Li S

Made substantial contributions to conception and design of the study, as well as provided administrative and technical support: Fowler N, Lavertu P, Teknos TN, Rezaee RP

\section{Availability of data and materials}

Not applicable.

\section{Financial support and sponsorship}

None.

\section{Conflicts of interest}

Dr. Rod P. Rezaee is a consultant for Zimer Biomet (Warsaw, IN). All other authors declared that there are no conflicts of interest.

\section{Ethical approval and consent to participate}

Not applicable.

\section{Consent for publication}

Not applicable.

\section{Copyright}

(c) The Author(s) 2021.

\section{REFERENCES}

1. Rosenthal E, Couch M, Farwell DG, Wax MK. Current concepts in microvascular reconstruction. Otolaryngol Head Neck Surg 2007;136:519-24. DOI PubMed

2. Kovatch KJ, Hanks JE, Stevens JR, Stucken CL. Current practices in microvascular reconstruction in otolaryngology-head and neck surgery. Laryngoscope 2019;129:138-45. DOI PubMed PMC

3. Thomas B, Warszawski J, Falkner F, et al. A comparative study of preoperative color-coded Duplex ultrasonography versus handheld 
audible Dopplers in ALT flap planning. Microsurgery 2020;40:561-7. DOI PubMed

4. Wade RG, Watford J, Wormald JCR, Bramhall RJ, Figus A. Perforator mapping reduces the operative time of DIEP flap breast reconstruction: a systematic review and meta-analysis of preoperative ultrasound, computed tomography and magnetic resonance angiography. J Plast Reconstr Aesthet Surg 2018;71:468-77. DOI PubMed

5. Garvey PB, Chang EI, Selber JC, et al. A prospective study of preoperative computed tomographic angiographic mapping of free fibula osteocutaneous flaps for head and neck reconstruction. Plast Reconstr Surg 2012;130:541e-9e. DOI PubMed PMC

6. Chow LC, Napoli A, Klein MB, Chang J, Rubin GD. Vascular mapping of the leg with multi-detector row CT angiography prior to free-flap transplantation. Radiology 2005;237:353-60. DOI PubMed

7. Rozen WM, Ashton MW, Stella DL, Phillips TJ, Taylor GI. Magnetic resonance angiography and computed tomographic angiography for free fibular flap transfer. J Reconstr Microsurg 2008;24:457-8. DOI PubMed

8. Chen SY, Lin WC, Deng SC, et al. Assessment of the perforators of anterolateral thigh flaps using 64-section multidetector computed tomographic angiography in head and neck cancer reconstruction. Eur J Surg Oncol 2010;36:1004-11. DOI PubMed

9. Garvey PB, Selber JC, Madewell JE, Bidaut L, Feng L, Yu P. A prospective study of preoperative computed tomographic angiography for head and neck reconstruction with anterolateral thigh flaps. Plast Reconstr Surg 2011;127:1505-14. DOI PubMed

10. Mun GH, Kim HJ, Cha MK, Kim WY. Impact of perforator mapping using multidetector-row computed tomographic angiography on free thoracodorsal artery perforator flap transfer. Plast Reconstr Surg 2008;122:1079-88. DOI PubMed

11. Kim JG, Lee SH. Comparison of the multidetector-row computed tomographic angiography axial and coronal planes' usefulness for detecting thoracodorsal artery perforators. Arch Plast Surg 2012;39:354-9. DOI PubMed PMC

12. Du E, Patel S, Huang B, Patel SN. Dual-phase CT angiography for presurgical planning in patients with vessel-depleted neck. Head Neck 2019;41:2929-36. DOI PubMed

13. Shen Y, Huang J, Dong MJ, Li J, Ye WM, Sun J. Application of computed tomography angiography mapping and located template for accurate location of perforator in head and neck reconstruction with anterolateral thigh perforator flap. Plast Reconstr Surg 2016;137:1875-85. DOI PubMed

14. Xiao W, Li K, Kiu-Huen Ng S, et al. A prospective comparative study of color doppler ultrasound and infrared thermography in the detection of perforators for anterolateral thigh flaps. Ann Plast Surg 2020;84:S190-5. DOI PubMed

15. Pereira N, Valenzuela D, Mangelsdorff G, Kufeke M, Roa R. Detection of perforators for free flap planning using smartphone thermal imaging: a concordance study with computed tomographic angiography in 120 perforators. Plast Reconstr Surg 2018;141:787-92. DOI PubMed

16. Tsuge I, Saito S, Sekiguchi H, et al. Photoacoustic tomography shows the branching pattern of anterolateral thigh perforators in vivo. Plast Reconstr Surg 2018;141:1288-92. DOI PubMed

17. Tsuge I, Saito S, Yamamoto G, et al. Preoperative vascular mapping for anterolateral thigh flap surgeries: a clinical trial of photoacoustic tomography imaging. Microsurgery 2020;40:324-30. DOI PubMed

18. Alkureishi L, Shaw-dunn J, Ross G. Effects of thinning the anterolateral thigh flap on the blood supply to the skin. Br J Plast Surg 2003;56:401-8. DOI PubMed

19. Kehrer A, Sachanadani NS, da Silva NPB, et al. Step-by-step guide to ultrasound-based design of alt flaps by the microsurgeon - basic and advanced applications and device settings. J Plast Reconstr Aesthet Surg 2020;73:1081-90. DOI PubMed

20. Martínez J, Torres Pérez A, Gijón Vega M, Nuñez-Villaveiran T. Preoperative vascular planning of free flaps: comparative study of computed tomographic angiography, color doppler ultrasonography, and hand-held doppler. Plast Reconstr Surg 2020;146:227-37. DOI PubMed

21. Cheng HT, Lin FY, Chang SC. Diagnostic efficacy of color Doppler ultrasonography in preoperative assessment of anterolateral thigh flap cutaneous perforators: an evidence-based review. Plast Reconstr Surg 2013;131:471e-3e. DOI PubMed

22. Nakayama K, Tamiya T, Yamamoto K, Akimoto S. A simple new apparatus for small vessel anastomosisi (free autograft of the sigmoid included). Surgery 1962;52:918-31. PubMed

23. Ostrup LT, Berggren A. The UNILINK instrument system for fast and safe microvascular anastomosis. Ann Plast Surg 1986;17:521-5. DOI PubMed

24. Maruccia M, Fatigato G, Elia R, et al. Microvascular coupler device versus hand-sewn venous anastomosis: a systematic review of the literature and data meta-analysis. Microsurgery 2020;40:608-17. DOI PubMed

25. Li R, Zhang R, He W, Qiao Y, Li W. The use of venous coupler device in free tissue transfers for oral and maxillofacial reconstruction. J Oral Maxillofac Surg 2015;73:2225-31. DOI PubMed

26. Assoumane A, Wang L, Liu K, Shang ZJ. Use of couplers for vascular anastomoses in 601 free flaps for reconstruction of defects of the head and neck: technique and two-year retrospective clinical study. Br J Oral Maxillofac Surg 2017;55:461-4. DOI PubMed

27. Zhang T, Lubek J, Salama A, et al. Venous anastomoses using microvascular coupler in free flap head and neck reconstruction. $J$ Oral Maxillofac Surg 2012;70:992-6. DOI PubMed

28. Frederick JW, Sweeny L, Carroll WR, Rosenthal EL. Microvascular anastomotic coupler assessment in head and neck reconstruction. Otolaryngol Head Neck Surg 2013;149:67-70. DOI PubMed PMC

29. Rozen WM, Whitaker IS, Acosta R. Venous coupler for free-flap anastomosis: outcomes of 1,000 cases. Anticancer Res 2010;30:1293-4. PubMed

30. DeLacure MD, Kuriakose MA, Spies AL. Clinical experience in end-to-side venous anastomoses with a microvascular anastomotic coupling device in head and neck reconstruction. Arch Otolaryngol Head Neck Surg 1999;125:869-72. DOI PubMed

31. Head LK, McKay DR. Economic comparison of hand-sutured and coupler-assisted microvascular anastomoses. J Reconstr Microsurg 2018;34:71-6. DOI PubMed 
32. Shindo ML, Costantino PD, Nalbone VP, Rice DH, Sinha UK. Use of a mechanical microvascular anastomotic device in head and neck free tissue transfer. Arch Otolaryngol Head Neck Surg 1996;122:529-32. DOI PubMed

33. Berggren A, Ostrup LT, Ragnarsson R. Clinical experience with the Unilink/3M Precise microvascular anastomotic device. Scand J Plast Reconstr Surg Hand Surg 1993;27:35-9. DOI PubMed

34. Ahn CY, Shaw WW, Berns S, Markowitz BL. Clinical experience with the $3 \mathrm{M}$ microvascular coupling anastomotic device in 100 freetissue transfers. Plast Reconstr Surg 1994;93:1481-4. DOI PubMed

35. Gundale AR, Berkovic YJ, Entezami P, Nathan CO, Chang BA. Systematic review of microvascular coupling devices for arterial anastomoses in free tissue transfer. Laryngoscope Investig Otolaryngol 2020;5:683-8. DOI PubMed PMC

36. Wain RA, Whitty JP, Dalal MD, Holmes MC, Ahmed W. Blood flow through sutured and coupled microvascular anastomoses: a comparative computational study. J Plast Reconstr Aesthet Surg 2014;67:951-9. DOI PubMed

37. Sando IC, Plott JS, McCracken BM, et al. Simplifying arterial coupling in microsurgery-a preclinical assessment of an everter device to aid with arterial anastomosis. J Reconstr Microsurg 2018;34:420-7. DOI PubMed

38. Spector JA, Draper LB, Levine JP, Ahn CY. Routine use of microvascular coupling device for arterial anastomosis in breast reconstruction. Ann Plast Surg 2006;56:365-8. DOI PubMed

39. Chernichenko N, Ross DA, Shin J, Chow JY, Sasaki CT, Ariyan S. Arterial coupling for microvascular free tissue transfer. Otolaryngol Head Neck Surg 2008;138:614-8. DOI PubMed

40. Li MM, Tamaki A, Seim NB, et al. Utilization of microvascular couplers in salvage arterial anastomosis in head and neck free flap surgery: case series and literature review. Head Neck 2020;42:E1-7. DOI PubMed

41. Belykh E, George L, Zhao X, et al. Microvascular anastomosis under 3D exoscope or endoscope magnification: a proof-of-concept study. Surg Neurol Int 2018;9:115. DOI PubMed PMC

42. Langer DJ, White TG, Schulder M, Boockvar JA, Labib M, Lawton MT. Advances in intraoperative optics: a brief review of current exoscope platforms. Oper Neurosurg (Hagerstown) 2020;19:84-93. DOI PubMed

43. Pinto V, Giorgini FA, Lozano Miralles ME, et al. 3D exoscope-assisted microvascular anastomosis: an evaluation on latex vessel models. J Clin Med 2020;9:3373. DOI PubMed PMC

44. Cheng HT, Ma H, Tsai CH, Hsu WL, Wang TH. A three-dimensional stereoscopic monitor system in microscopic vascular anastomosis. Microsurgery 2012;32:571-4. DOI PubMed

45. Piatkowski AA, Keuter XHA, Schols RM, van der Hulst RRWJ. Potential of performing a microvascular free flap reconstruction using solely a 3D exoscope instead of a conventional microscope. J Plast Reconstr Aesthet Surg 2018;71:1664-78. DOI PubMed

46. Ichikawa Y, Senda D, Shingyochi Y, Mizuno H. Potential advantages of using three-dimensional exoscope for microvascular anastomosis in free flap transfer. Plast Reconstr Surg 2019;144:726e-7e. DOI PubMed

47. De Virgilio A, Mercante G, Gaino F, et al. Preliminary clinical experience with the 4 K3-dimensional microvideoscope (VITOM 3D) system for free flap head and neck reconstruction. Head Neck 2020;42:138-40. DOI PubMed

48. De Virgilio A, Iocca O, Di Maio P, et al. Free flap microvascular anastomosis in head and neck reconstruction using a $4 \mathrm{~K}$ threedimensional exoscope system (VITOM 3D). Int J Oral Maxillofac Surg 2020;49:1169-73. DOI PubMed

49. Molteni G, Nocini R, Ghirelli M, et al. Free flap head and neck microsurgery with VITOM ${ }^{\circledR}$ 3D: Surgical outcomes and surgeon's perspective. Auris Nasus Larynx 2021;48:464-70. DOI PubMed

50. Ahmad FI, Mericli AF, DeFazio MV, et al. Application of the ORBEYE three-dimensional exoscope for microsurgical procedures. Microsurgery 2020;40:468-72. DOI PubMed

51. Yeoh MS, Kim DD, Ghali GE. Fluorescence angiography in the assessment of flap perfusion and vitality. Oral Maxillofac Surg Clin North Am 2013;25:61-6, vi. DOI PubMed

52. Holzbach T, Artunian N, Spanholtz TA, Volkmer E, Engelhardt TO, Giunta RE. Microscope-integrated intraoperative indocyanine green angiography in plastic surgery. Handchir Mikrochir Plast Chir 2012;44:84-8. DOI PubMed

53. Hope-Ross M, Yannuzzi LA, Gragoudas ES, et al. Adverse reactions due to indocyanine green. Ophthalmology 1994;101:529-33. DOI PubMed

54. Chu W, Chennamsetty A, Toroussian R, Lau C. Anaphylactic shock after intravenous administration of indocyanine green during robotic partial nephrectomy. Urol Case Rep 2017;12:37-8. DOI PubMed PMC

55. Wax MK. The role of the implantable Doppler probe in free flap surgery. Laryngoscope 2014;124 Suppl 1:S1-12. DOI PubMed

56. Chang EI, Chu CK, Chang EI. Advancements in imaging technology for microvascular free tissue transfer. J Surg Oncol 2018;118:729-35. DOI PubMed

57. Chang TY, Lee YC, Lin YC, et al. Implantable doppler probes for postoperatively monitoring free flaps: efficacy. A systematic review and meta-analysis. Plast Reconstr Surg Glob Open 2016;4:e1099. DOI PubMed PMC

58. Swartz WM, Izquierdo R, Miller MJ. Implantable venous Doppler microvascular monitoring: laboratory investigation and clinical results. Plast Reconstr Surg 1994;93:152-63. DOI PubMed

59. Guillemaud JP, Seikaly H, Cote D, Allen H, Harris JR. The implantable Cook-Swartz Doppler probe for postoperative monitoring in head and neck free flap reconstruction. Arch Otolaryngol Head Neck Surg 2008;134:729-34. DOI PubMed

60. Seres L, Makula E, Morvay Z, Borbely L. Color Doppler ultrasound for monitoring free flaps in the head and neck region. J Craniofac Surg 2002;13:75-8. DOI PubMed

61. Vakharia KT, Henstrom D, Lindsay R, Cunnane MB, Cheney M, Hadlock T. Color Doppler ultrasound: effective monitoring of the buried free flap in facial reanimation. Otolaryngol Head Neck Surg 2012;146:372-6. DOI PubMed

62. Rosenberg JJ, Fornage BD, Chevray PM. Monitoring buried free flaps: limitations of the implantable Doppler and use of color duplex sonography as a confirmatory test. Plast Reconstr Surg 2006;118:109-13; discussion 114. DOI PubMed 
63. Repez A, Oroszy D, Arnez ZM. Continuous postoperative monitoring of cutaneous free flaps using near infrared spectroscopy. J Plast Reconstr Aesthet Surg 2008;61:71-7. DOI PubMed

64. Chen Y, Shen Z, Shao Z, Yu P, Wu J. Free flap monitoring using near-infrared spectroscopy: a systemic review. Ann Plast Surg 2016;76:590-7. DOI PubMed

65. Steele MH. Three-year experience using near infrared spectroscopy tissue oximetry monitoring of free tissue transfers. Ann Plast Surg 2011;66:540-5. DOI PubMed

66. Svensson H, Pettersson H, Svedman P. Laser Doppler flowmetry and laser photometry for monitoring free flaps. Scand J Plast Reconstr Surg 1985;19:245-9. DOI PubMed

67. Yuen JC, Feng Z. Monitoring free flaps using the laser Doppler flowmeter: five-year experience. Plast Reconstr Surg 2000;105:55-61. DOI PubMed

68. Clinton MS, Sepka RS, Bristol D, et al. Establishment of normal ranges of laser Doppler blood flow in autologous tissue transplants. Plast Reconstr Surg 1991;87:299-309. DOI PubMed

69. Hölzle F, Loeffelbein DJ, Nolte D, Wolff KD. Free flap monitoring using simultaneous non-invasive laser Doppler flowmetry and tissue spectrophotometry. J Craniomaxillofac Surg 2006;34:25-33. DOI PubMed

70. Kannan RY. Early detection of inflow problems during free flap monitoring using digital tympanic thermometers. $J$ Plast Reconstr Aesthet Surg 2012;65:e135. DOI PubMed

71. Khouri RK, Shaw WW. Monitoring of free flaps with surface-temperature recordings: is it reliable? Plast Reconstr Surg 1992;89:4959; discussion 500. PubMed

72. Baudet J, LeMaire JM, Guimberteau JC. Ten free groin flaps. Plast Reconstr Surg 1976;57:577-95. DOI PubMed

73. Papillion P, Wong L, Waldrop J, et al. Infrared surface temperature monitoring in the postoperative management of free tissue transfers. Can J Plast Surg 2009;17:97-101. DOI PubMed PMC

74. Perng CK, Ma H, Chiu YJ, Lin PH, Tsai CH. Detection of free flap pedicle thrombosis by infrared surface temperature imaging. $J$ Surg Res 2018;229:169-76. DOI PubMed 